\title{
Robotic Assisted Laparoscopic Treatment of Gonadal Vein Syndrome in a Boy
}

\author{
Hubert S. Swana, Alejandro R. Rodriguez, Timothy A. Kim, Mark A. Rich \\ Nemours Children's Clinic Orlando, Florida, USA and Department of Urology, University of South \\ Florida School of Medicine, Tampa, Florida, USA
}

\begin{abstract}
Purpose: Gonadal vein syndrome, with ureteral obstruction and compression by an overlying testicular vein is a controversial and rare diagnosis. Open, laparoscopic, and robot-assisted laparoscopic repairs have been described. We report the first case of robot-assisted gonadal vein ligation for treatment of gonadal vein syndrome in a nine year-old boy.

Materials and Methods: A 9 years-old boy presented with a four to six month history of worsening intermittent flank pain, nausea and vomiting. Ultrasound revealed moderate hydronephrosis. Diuretic renography and intravenous pyelography reproduced his pain and demonstrated left-sided hydronephrosis and obstruction. The patient underwent left robot-assisted surgery via a four port approach. The colon was reflected medially. The gonadal vein was dissected off the underlying ureter and ligated using laparoscopic clips. Segmental vein excision and ureterolysis was performed. Inspection of the renal hilum did not reveal any other crossing vessels. Results: Operative time was 94 minutes. The patient was discharged 36 hours after surgery. His hydronephrosis has resolved completely. He remains pain-free nine months after surgery.

Conclusion: Robot-assisted laparoscopic vein excision and ureterolysis is a safe option for the management of ureteral obstruction caused by the gonadal vein.
\end{abstract}

Int Braz J Urol. 2011; 37 (Video \#1): 134_5

Available at: www.brazjurol.com.br/videos/january_february_2011/Swana_134_135video.htm

\section{Correspondence address:}

Dr. Hubert S. Swana

Nemours Children's Clinic Orlando

1717 S. Orange Ave, Suite 100

Orlando, Florida, 32806, USA

E-mail: hswana@nemours.org 


\section{EDITORIAL COMMENT}

In this video by Swana et al., a novel application of robotic assisted laparoscopic surgery to pediatric urology is very well depicted. This case of gonadal vein syndrome in a symptomatic 9 year old boy highlights that the superior three-dimensional vision and surgical dexterity/range of motion attributable to current robotic surgical technology is in some ways ideally suited to the surgical management of pediatric urological conditions. As with all evolving surgical technologies, patient / case selection remains the one pivotal criterion best predicting treatment related outcome. I once again applaud these authors on a very illustrative and innovative surgical approach to a case of gonadal vein syndrome.

Dr. Philippe E. Spiess Assistant Professor of Urologic Oncology H. Lee Moffitt Cancer Center Tampa, Florida, USA E-mail:Philippe.Spiess@moffitt.org 